\title{
Categorical sequences
}

\author{
ROB NENDORF \\ NiCK SCOVILLE \\ JEFF STROM
}

\begin{abstract}
We define and study the categorical sequence of a space, which is a new formalism that streamlines the computation of the Lusternik-Schnirelmann category of a space $X$ by induction on its CW skeleta. The $k^{\text {th }}$ term in the categorical sequence of a CW complex $X, \sigma_{X}(k)$, is the least integer $n$ for which cat ${ }_{X}\left(X_{n}\right) \geq k$. We show that $\sigma_{X}$ is a well-defined homotopy invariant of $X$. We prove that $\sigma_{X}(k+l) \geq \sigma_{X}(k)+\sigma_{X}(l)$, which is one of three keys to the power of categorical sequences. In addition to this formula, we provide formulas relating the categorical sequences of spaces and some of their algebraic invariants, including their cohomology algebras and their rational models; we also find relations between the categorical sequences of the spaces in a fibration sequence and give a preliminary result on the categorical sequence of a product of two spaces in the rational case. We completely characterize the sequences which can arise as categorical sequences of formal rational spaces. The most important of the many examples that we offer is a simple proof of a theorem of Ghienne: if $X$ is a member of the Mislin genus of the Lie group $S p(3)$, then $\operatorname{cat}(X)=\operatorname{cat}(S p(3))$.

55M30; 55P62
\end{abstract}

\section{Introduction}

The Lusternik-Schnirelmann category of a topological space $X$ is the least integer $k$ for which $X$ has an open cover $X=X_{0} \cup X_{1} \cup \cdots \cup X_{k}$ with the property that each inclusion map $X_{j} \hookrightarrow X$ is homotopic to a constant map; it is denoted cat $(X)$. This homotopy invariant of topological spaces was first introduced by Lusternik and Schnirelmann in 1934 as a tool to use in studying functions on (compact) manifolds: a smooth function $f: M \rightarrow \mathbb{R}$ must have at least cat $(M)+1$ critical points.

If $X$ is a CW complex, then $X_{n}=X_{n-1} \cup_{\alpha}$ (n-cells), and therefore cat $\left(X_{n}\right) \leq$ $\operatorname{cat}\left(X_{n-1}\right)+1$. Berstein and Hilton asked [3] what conditions must be placed on the attaching map $\alpha$ in order to guarantee that equality holds in this upper bound; the answer is that equality holds when a certain set of generalized Hopf invariants does 
not contain the trivial map $*$. Thus it is possible, at least in principle, to compute the Lusternik-Schnirelmann category of a finite-dimensional CW complex inductively up its skeleta.

It was shown by the third author [34] that the Hopf sets for lower-dimensional skeleta partially determine the Hopf sets for high-dimensional skeleta. In actual computations, this makes it possible to 'bootstrap' up from relatively simple low-dimensional results to (apparently) difficult high-dimensional calculations. Our goal in this paper is to establish a convenient formalism for doing category calculations while making use of all low-dimensional information.

This is done via the categorical sequence of a space $X$, which is a function $\sigma_{X}: \mathbb{N} \rightarrow$ $\mathbb{N} \cup\{\infty\}$ defined by

$$
\sigma_{X}(k)=\inf \left\{n \mid \operatorname{cat}_{X}\left(X_{n}\right) \geq k\right\}
$$

where $\operatorname{cat}_{X}\left(X_{n}\right)$ is the category of $X_{n}$ relative to $X$ (see Definition 4) ${ }^{1}$. It is shown in Propositions 2.1 and 2.2 that $\sigma_{X}$ is a well-defined homotopy invariant of $X$; ie, when $n$ is larger than the connectivity of $X$, $\operatorname{cat}_{X}\left(X_{n}\right)$ depends only on $n$ and the homotopy type of $X$, and not on any choices made in constructing a CW decomposition of $X$. If $X$ is finite-dimensional, then $\sigma_{X}$ determines cat $(X)$; examples due to Roitberg [28] show that this is not true for infinite-dimensional spaces. In any case, the categorical sequence of $X$ holds a wealth of useful information.

Though we are not directly concerned with the applications of Lusternik-Schnirelmann category to critical point theory in this paper, categorical sequences could play a useful role there. For example, in the study of the $n$-body problem, one is often interested in infinite-dimensional Sobolev spaces $W$; in order to apply the Lusternik-Schnirelmann method in this situation, it is necessary to find compact subsets $K \subseteq W$ such that the relative category $\operatorname{cat}_{W}(K)$ is large (see Ambrosetti and Zelati [1, Remarks 2.15 and 3.5], Fadell and Husseini [8, Theorem 4.6], or Rabinowitz [27], for example). The categorical sequence of $W$ gives lower estimates on the dimension of such subsets. If $\sigma_{W}(k)=n$, then cat ${ }_{W}\left(W_{n-1}\right)<k$; if $\operatorname{dim}(K)<n$ then $K$ can be deformed into $W_{n-1}$, and so cat $_{W}(K)<k$.

Our theoretical results establish formulas for calculation with categorical sequences. Some of the statements make use of another sequence, the product length sequence of a non-negatively graded commutative algebra $\mathcal{A}$, defined by setting $\sigma_{\mathcal{A}}(k)$ to be

\footnotetext{
${ }^{1}$ In his encyclopedic paper [15], Fox defined a categorical sequence to be a certain kind of filtration of a space; this idea was used in his proof of the product inequality cat $(X \times Y) \leq \operatorname{cat}(X)+\operatorname{cat}(Y)$. Our use of the term 'categorical sequence' for a completely different idea should carry no risk of confusion, since the earlier notion is no longer used, at least in the homotopy theory of Lusternik-Schnirelmann category (but see Cicortaş $[5 ; 6]$ for an equivariant version).
} 
the least dimension $n$ for which the $n^{\text {th }}$ grading $\mathcal{A}^{n}$ of $\mathcal{A}$ contains a nontrivial $k$-fold product.

Proposition 3.2 For any space $X$ and any ring $R, \sigma_{X} \leq \sigma_{H^{*}(X ; R)}$.

We also estimate the categorical sequence of a rational space in terms of any of its models.

Proposition 3.7 If $X$ is a simply-connected rational space and $\mathcal{A}$ is any model for $X$, then $\sigma_{X} \geq \sigma_{\mathcal{A}}$.

Recall that a simply-connected rational space $X$ is formal if its cohomology algebra $H^{*}(X)$, with trivial differential, is a model for $X$. Thus we have the following computation for formal spaces.

Proposition 5.1 If $X$ is a simply-connected formal rational space, then $\sigma_{X}=\sigma_{H^{*}(X)}$.

More generally, we completely determine the sequences $\sigma$ which can arise as the categorical sequences of simply-connected formal rational spaces.

Theorem 5.2 The following conditions on a sequence $\sigma$ with $\sigma(1)>1$ are equivalent:

(a) $\sigma=\sigma_{\mathcal{A}}$ for some commutative graded algebra $\mathcal{A}$,

(b) $\sigma(k+1) \geq \frac{k+1}{k} \sigma(k)$ for each $k$,

(c) $\sigma=\sigma_{W}$ where $W=\bigvee P_{i}$ and $P_{i}=\prod S^{n_{j}}$ is a product of spheres, and

(d) $\sigma=\sigma_{X}$ for some formal rational space $X$.

The keys to the computational power of categorical sequences, though, are the three properties listed in the following theorem. In order to prove parts (b) and (c) for all spaces (and not just spaces of finite type, say), we have to use a set-theoretical framework in which Whitehead's Problem (which asks: $\operatorname{does} \operatorname{Ext}(A, \mathbb{Z})=0$ imply $A$ is free?) has a positive solution. See Section 1.1 and Lemma 1.3 for details.

Theorem 3.4 For any space $X$,

(a) $\sigma_{X}(k+l) \geq \sigma_{X}(k)+\sigma_{X}(l)$,

(b) if $X$ is simply-connected and $\sigma_{X}(k)=n$, then $H^{n}(X ; A) \neq 0$ for some coeffcient group $A$, and 
(c) if equality occurs in (a) and X is simply-connected, then the cup product

$$
H^{k}(X ; A) \otimes H^{l}(X ; B) \rightarrow H^{k+l}(X ; A \otimes B)
$$

is nontrivial for some choice of coefficients.

The point we hope to make in this paper is that calculation with sequences is no harder than calculation of category; indeed, the extra information contained in the sequence, together with Theorem 3.4, can greatly facilitate computations. To illustrate this point, let $X$ be any simply-connected space such that $H^{*}(X ; \mathbb{Z}) \cong H^{*}(\operatorname{Sp}(3) ; \mathbb{Z})=$ $\Lambda\left(x_{3}, x_{7}, x_{11}\right)$. The categorical sequence $\sigma_{X}$ clearly has $\sigma_{X}(1)=3$ and $\sigma_{X}(2) \geq 7$ by Theorem 3.4(b). By Theorem 3.4(a), $\sigma_{X}(4) \geq \sigma_{X}(2)+\sigma_{X}(2) \geq 14$. Furthermore, $\sigma_{X}(4)>14$ by Theorem 3.4(c), because the cup product $H^{7}(X) \otimes H^{7}(X) \rightarrow H^{14}(X)$ is trivial. Now we have $\sigma_{X}(4) \geq 18$ by Theorem 3.4(b), and hence $\sigma_{X}(5) \geq \sigma_{X}(4)+$ $\sigma_{X}(1)=21$ by Theorem 3.4(a). Since $X$ is 21-dimensional (up to homotopy), this proves that $\operatorname{cat}(X) \leq 5$ by Theorem $3.4($ b). We will see in Theorem 7.1 below that this calculation constitutes a simple proof of a result of Ghienne [18] about the Mislin genus of $S p(3)$.

Theorem 3.4 can also be used to prove a generalization of a somewhat obscure result of Ganea [17].

Corollary 3.6 Let $X$ be simply-connected and of finite type with $\sigma_{X}(k)=n$. If there are integers $0<a_{1}<a_{2}<\cdots<a_{l}$ such that

$$
\left\{n \mid \widetilde{H}^{n}(X ; G) \neq 0 \text { for some } G\right\} \subseteq I_{1} \cup I_{2} \cup \cdots \cup I_{l}
$$

where $I_{j}=\left[a_{j}, a_{j}+(n-1)\right]$ (brackets denote closed intervals in $\left.\mathbb{R}\right)$, then cat $(X)<$ $k(l+1)$.

The importance of Corollary 3.6 is not the result as such. Rather, it is the fact that, since it simply encodes an elementary computation with sequences, the result can be safely disregarded without losing computational power. Our proof is completely different from the one given in [17]. Ganea's proof makes use of the Blakers-Massey theorem: certain cofiber sequences are treated as fibration sequences. Our argument uses Theorem 3.4, which in turn rests on a much more elementary fact: the factorization $\Delta_{k+l}=\left(\Delta_{k} \times \Delta_{l}\right) \circ \Delta_{2}$ of diagonal maps. However, Ganea's theorem also applies to the strong category of $X$, while ours only works for ordinary category.

One of our most pleasing general results gives formulas relating the categorical sequences of the spaces in a fibration sequence. 
Theorem 4.2 Let $F \stackrel{q}{\longrightarrow} E \stackrel{p}{\longrightarrow} B$ be a fibration sequence and write $a=\operatorname{cat}(q) \leq$ $\operatorname{cat}(F)$ and $b=\operatorname{cat}(p) \leq \operatorname{cat}(B)$. Then

(a) $\sigma_{E}(k(a+1)) \geq \sigma_{B}(k)$, and

(b) $\sigma_{E}(k(b+1)) \geq \sigma_{F}(k)$.

As a corollary to Theorem 4.2 we obtain the following elaboration of the celebrated Mapping Theorem from the rational theory of Lusternik-Schnirelmann category.

Proposition 4.7 Let $f: X \rightarrow Y$ be a map between simply-connected rational spaces which induces an injective map $f_{*}: \pi_{*}(X) \rightarrow \pi_{*}(Y)$. Then $\sigma_{X} \geq \sigma_{Y}$.

Finally, we address products. To state our result (and our conjectures), we construct, for sequences $\sigma$ and $\tau$, a 'product sequence' $\sigma * \tau$ defined by $\sigma * \tau(k)=\min \{\sigma(i)+$ $\tau(j) \mid i+j=k\}$. It is not hard to see, using Proposition 3.7, that if $X$ and $Y$ are simply-connected formal rational spaces, then $\sigma_{X \times Y}=\sigma_{X} * \sigma_{Y}$. We conjecture that this equation holds in general for simply-connected rational spaces. So far however, the best we have been able to do is an inequality.

Theorem 6.2 For simply-connected rational $X$ and $Y, \sigma_{X \times Y} \leq \sigma_{X} * \sigma_{Y}$.

This inequality is certainly not true in general for non-rational spaces, as the examples of Iwase [22] show. However, we conjecture that the reverse inequality $\sigma_{X} * \sigma_{Y} \leq \sigma_{X \times Y}$ is valid, not only for rational spaces, but for all spaces.

Acknowledgements We thank Don Stanley, Martin Arkowitz, Yves Félix, Daniel Tanré and Terrell Hodge for their interest and advice at various stages of this project.

\section{Preliminaries}

In this section we establish the basic notation and concepts that will be used in the body of the paper.

\subsection{Basics}

We work with pointed spaces and maps; we use $*$ to denote the one point space or any trivial map. We use $\operatorname{id}_{X}: X \rightarrow X$ to denote the identity map and $\Delta_{k}: X \rightarrow X^{k}$ to denote the diagonal map $\Delta_{k}(x)=(x, x, \ldots, x)$. The symbol $\simeq$ denotes homotopy equivalence of spaces or homotopy of maps. All solid arrow diagrams in this paper are (homotopy) commutative.

If $S$ is a set of real numbers, then $\inf (S)$ is the infimum of $S$. We adopt the usual convention that $\inf (\varnothing)=\infty$. 
Set theory Whitehead's Problem asks: if $A$ is an abelian group such that $\operatorname{Ext}(A, \mathbb{Z})=$ 0 , does it follow that $A$ is free? The answer is 'yes' if $A$ is finitely generated. Shelah has shown that the general problem is undecidable in ordinary ZFC set theory, but the answer is 'yes' if Gödel's Axiom of Constructibility is assumed (see Shelah [32]). In order to avoid 'unnecessary' hypotheses in Lemma 1.3 and Theorem 3.4 below,

$\star \quad$ we work in a set theory where $\operatorname{Ext}(A, \mathbb{Z})=0$ implies that $A$ is free.

For those uncomfortable with this assumption, we emphasize that ordinary ZFC set theory is sufficient to prove Lemma 1.3 and Theorem 3.4 when $H^{n}(X ; \mathbb{Z})$ is finitely generated for each $n$.

\subsection{Skeleta}

We are concerned with the Lusternik-Schnirelmann category of the CW skeleta of a space. It will simplify some of our later work to use the following slightly abstract notion of skeleton.

Definition 1.1 An $n$-skeleton for a space $X$ is a map $i: X_{n} \rightarrow X$, where $X_{n}$ is a CW complex such that

(a) $X_{n}$ is $n$-dimensional (up to homotopy), and

(b) $i$ is an $n$-equivalence.

This definition is justified by the observation that an $n$-skeleton $i: X_{n} \rightarrow X$ can be taken as the $n^{\text {th }} \mathrm{CW}$ skeleton of a CW replacement for $X$.

The following result will help us to recognize skeleta. We omit the proof.

Lemma 1.2 Let $i: A \rightarrow X$ where $A$ and $X$ are simply-connected. Then $i$ is an $n$-skeleton for $X$ if and only if

(a) $H^{*}(A)=0$ for $*>n$ in all coefficients, and

(b) the induced map $i^{*}: H^{*}(X) \rightarrow H^{*}(A)$ is an isomorphism for $*<n$ and is injective for $*=n$ in all coefficients.

When we are working with rational spaces (see Félix, Halperin and Thomas [11]) we will want our skeleta to also be rational spaces. Unfortunately, this won't always happen; for example, the inclusion $\bigvee_{n=1}^{\infty} S^{n} \hookrightarrow S_{\mathbb{Q}}^{n}$ is an $n$-skeleton for the rational $n$-sphere. We avoid this problem by defining a rational $n$-skeleton of a simply-connected rational space $X$ to be a map $i: X_{n} \rightarrow X$ where $X_{n}$ is a simply-connected rational space such that

Algebraic $8 \mathcal{G}$ Geometric Topology, Volume 6 (2006) 
(a) $H^{*}\left(X_{n} ; \mathbb{Q}\right)=0$ for $*>n$, and

(b) the induced map $i^{*}: H^{*}(X ; \mathbb{Q}) \rightarrow H^{*}\left(X_{n} ; \mathbb{Q}\right)$ is an isomorphism for $*<n$ and is injective for $*=n$.

Rational $n$-skeleta are plentiful: if $X$ is a rational space and $X_{n}$ is an (integral) $n$-skeleton of $X$, then $\left(X_{n}\right)_{\mathbb{Q}}$ is a rational $n$-skeleton of $X$. We make a standing convention that if a space $X$ is assumed to be rational, then whenever we refer to an $n$-skeleton of $X$, we actually mean a rational $n$-skeleton.

The proof of part (b) of our next result in full generality depends on a positive solution to Whitehead's Problem.

Lemma 1.3 Let $X$ be a simply-connected space.

(a) If $H_{n}(X ; \mathbb{Z})$ is free abelian, then $X$ has an $n$-skeleton $i: X_{n} \rightarrow X$ such that $i^{*}: H^{*}(X) \rightarrow H^{*}\left(X_{n}\right)$ is an isomorphism for $* \leq n$,

(b) If $H^{n}(X ; A)=0$ for all coefficient groups $A$, then $X$ has an $(n-1)$-dimensional $n$-skeleton.

The corresponding statements also hold for all simply-connected rational spaces.

Proof Write $M(G, n)$ for the Moore space with

$$
H_{n}(M(G, n) ; \mathbb{Z}) \cong G \quad \text { and } \quad H_{i}(M(G, n) ; \mathbb{Z})=0 \quad \text { for } i \neq 0, n .
$$

When $G$ is free abelian, we take $M(G, n)=\bigvee S^{n}$. According to Brown and Copeland [4], any simply-connected space $X$ admits a homology decomposition, ie, a sequence of $\mathrm{CW}$ complexes $X(n)$ which are related to one another by cofiber sequences $M_{n-1} \rightarrow X(n-1) \rightarrow X(n)$ (where $\left.M_{n-1}=M\left(H_{n}(X ; \mathbb{Z}), n-1\right)\right)$ and satisfy $X \simeq \operatorname{hocolim}_{n} X(n)$. The inclusion map $X(n) \rightarrow X$ induces isomorphisms on integral homology through dimension $n$, and $H_{k}(X(n) ; \mathbb{Z})=0$ for $k>n$.

With the CW decomposition inherited from the colimit, $X_{n} \subseteq X(n) \subseteq X_{n+1}$ for each $n$. If $H_{n}(X ; \mathbb{Z})$ is free abelian then $X(n)=X(n-1) \cup(n-$ cells $)$, so $X(n)$ is $n$-dimensional and hence $X_{n}=X(n)$. Thus $X(n)$ is the desired $n$-skeleton of $X$.

To prove (b), assume that $H^{n}(X ; A)=0$ for all $A$. Using the Universal Coefficient isomorphism (see Switzer [35, Corollary 13.11]), we obtain

$$
\operatorname{Hom}\left(H_{n}(X ; \mathbb{Z}), A\right)=0 \quad \text { and } \quad \operatorname{Ext}\left(H_{n-1}(X ; \mathbb{Z}) ; A\right)=0
$$

for all $A$. We claim that (i) $H_{n}(X ; \mathbb{Z})=0$ and (ii) $H_{n-1}(X ; \mathbb{Z})$ is free abelian. To prove (i), we let $A=H_{n}(X ; \mathbb{Z})$; if $A$ were nonzero, then $\operatorname{Hom}\left(H_{n}(X ; \mathbb{Z}), A\right)$ would 
be nonzero (since it contains the identity map), thereby contradicting the assumption. For (ii), we set $A=\mathbb{Z}$; now $\operatorname{Ext}\left(H_{n-1}(X ; \mathbb{Z}), \mathbb{Z}\right)=0$, and by Whitehead's Problem, we conclude that $H_{n-1}(X ; \mathbb{Z})$ is free.

Now apply part (a) to conclude that $X(n-1)$ is an $(n-1)$-dimensional $(n-1)$-skeleton and $X(n)$ is an $n$-skeleton of $X$. Since $H_{n}(M(G, n) ; \mathbb{Z})=0$, we have $M_{n-1} \simeq *$, so $X(n) \simeq X(n-1)$, and hence $X(n)$ is an $(n-1)$-dimensional $n$-skeleton for $X$.

\subsection{Lusternik-Schnirelmann category}

We make use of three equivalent definitions of the Lusternik-Schnirelmann category of maps and spaces.

Definition 1.4 The Lusternik-Schnirelmann category of a map $f: X \rightarrow Y$ is the least integer $k$ for which $X$ has a cover by open sets

$$
X=X_{0} \cup X_{1} \cup \cdots \cup X_{k}
$$

such that $\left.f\right|_{X_{i}} \simeq *$ for each $i$. When $f=\operatorname{id}_{X}$, we write $\operatorname{cat}(X)=\operatorname{cat}\left(\operatorname{id}_{X}\right)$ and when $i: A \hookrightarrow X$, we write $\operatorname{cat}_{X}(A)=\operatorname{cat}(i)$.

If $X$ is a CW complex, then it is equivalent to require each $X_{i}$ to be a subcomplex of $X$ in some CW decomposition.

The category of $f: X \rightarrow Y$ can also be defined in terms of the Ganea fibrations $p_{k}: G_{k}(Y) \rightarrow Y$ with fiber $F_{k}(Y)$. The inductive definition of these fibrations begins by defining

$$
F_{0}(Y) \longrightarrow G_{0}(Y) \stackrel{p_{0}}{\longrightarrow} Y
$$

to be the familiar path-loop fibration sequence

$$
\Omega(Y) \longrightarrow \mathcal{P}(Y) \longrightarrow Y .
$$

Given the $k^{\text {th }}$ Ganea fibration sequence

$$
F_{k}(Y) \longrightarrow G_{k}(Y) \stackrel{p_{k}}{\longrightarrow} Y,
$$

let $\bar{G}_{k+1}(Y)=G_{k}(Y) \cup C F_{k}(Y)$ be the cofiber of $p_{k}$ and define $\bar{p}_{k+1}: \bar{G}_{k+1}(Y) \rightarrow$ $Y$ by sending the cone to the base point of $Y$. The $(k+1)^{\text {st }}$ Ganea fibration $p_{k+1}: G_{k+1}(Y) \rightarrow Y$ results from converting the map $\bar{p}_{k+1}$ to a fibration. A result of Ganea [17] implies that $\operatorname{cat}(f) \leq k$ if and only if there is a lift $\lambda$ of $f$ in the diagram

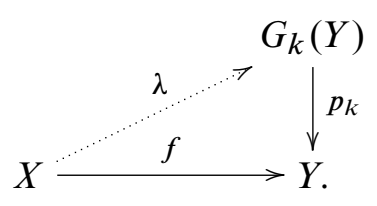

Algebraic 83 Geometric Topology, Volume 6 (2006) 
Our third definition is due to $\mathrm{GW}$ Whitehead. According to [36, page 458], cat $(f) \leq k$ if and only if the composition of $f$ with the diagonal map of pairs

$$
(X, *) \stackrel{f}{\longrightarrow}(Y, *) \stackrel{\Delta_{k+1}}{\longrightarrow}(Y, *)^{k+1}
$$

factors, up to homotopy of pairs, through the trivial pair $(X, X)$.

We will make use of a related invariant, called Qcat, which is defined in terms of the fibrations that result from applying a fiberwise version of the infinite suspension functor $Q$ to the Ganea fibrations. Let $q_{k}: \widetilde{G}_{k}(Y) \rightarrow Y$ denote the fiberwise infinite suspension of the $k$-th Ganea fibration. Then Qcat $(f)$ is the least integer $k$ for which $f$ lifts through $q_{k}$ (see Scheerer, Stanley and Tanré [29]).

\subsection{Rational homotopy and Lusternik-Schnirelmann category}

We briefly recall some key elements of the rational theory of Lusternik-Schnirelmann category. The reader is encouraged to consult Cornea, Lupton, Oprea and Tanré [7, Chapter 5] or Félix, Halperin and Thomas [11, Part V] for details.

A (simply-connected) Sullivan algebra is a commutative differential graded algebra (CDGA) $\mathcal{A}$ over $\mathbb{Q}$ such that: (a) $\mathcal{A}^{0} \cong \mathbb{Q}$ and $\mathcal{A}^{1}=0$; (b) as a $\mathbb{Q}$-algebra, $\mathcal{A} \cong \Lambda(V)$ where $V$ is a graded vector space; and (c) the differential $d$ is decomposable in the sense that $d(\mathcal{A}) \subseteq \overline{\mathcal{A}}^{2}$, where $\overline{\mathcal{A}}$ is the augmentation ideal of $\mathcal{A}$. Every simplyconnected space $X$ has a Sullivan minimal model, $\mathcal{M}(X)$, which is a Sullivan algebra such that $H^{*}(\mathcal{M}(X)) \cong H^{*}(X ; \mathbb{Q})$. A model for $X$ is any CDGA for which there is a map $\phi: \mathcal{M}(X) \rightarrow \mathcal{A}$ which induces an isomorphism in cohomology ( $\phi$ is a quasi-isomorphism).

Definition 1.5 Let $\mathcal{A}$ be an augmented CDGA and write $\overline{\mathcal{A}}$ for the augmentation ideal. The nilpotency of $A$, denoted nil $(\mathcal{A})$, is the greatest integer $k$ such that $(\overline{\mathcal{A}})^{k} \neq 0$.

The algebraic study of the Lusternik-Schnirelmann category of rational spaces can be developed from the following result, which can be found in [7, Corollary 5.16] (though, historically, it was not [7, Remark 5.15]).

Theorem 1.6 If $X$ is a rational space, then the following are equivalent

(a) $\operatorname{cat}(X) \leq k$, and

(b) $\mathcal{M}(X)$ is a retract (up to chain homotopy) of a Sullivan algebra $\mathcal{B}$ which is quasi-isomorphic with another $C D G A \mathcal{A}$ with $\operatorname{nil}(\mathcal{A}) \leq k$. 
It follows immediately from Theorem 1.6 that if $u \in H^{*}(Y)=H^{*}(\mathcal{M}(Y))$ can be represented by a cocycle which is a $k$-fold product, then $f^{*}(u)=0$ for any map $f: X \rightarrow Y$ with $\operatorname{cat}(X)<k$. In this case, the (rational) category weight of $u$ is at least $k$. We write $\operatorname{wgt}(u) \geq k$ and observe that $\operatorname{cat}(X) \geq \operatorname{wgt}(u)$ whenever $u \neq 0 \in H^{*}(X)$. The maximum value of $\operatorname{wgt}(u)$ for $u \in H^{*}(X)$ is known as the Toomer invariant of $X$, and is denoted $e_{0}(X)$.

There is a related invariant, denoted Mcat (see Félix [9]). It is known that $\operatorname{Mcat}(X)=$ cat $(X)$ for simply-connected rational spaces (see Hess [21, Theorem 0]). The equality of Mcat and cat is known to fail for maps: according to Parent [26, Theorems 2 and 11] $\operatorname{Mcat}(f \times g)=\operatorname{Mcat}(f)+\operatorname{Mcat}(g)$; on the other hand, Stanley [33] has produced examples of maps $f$ and $g$ between simply-connected rational spaces such that $\operatorname{cat}(f \times g)<\operatorname{cat}(f)+\operatorname{cat}(g)$. It is also known that $\operatorname{Mcat}(X)=\operatorname{Qcat}(X)$ when $X$ is a simply-connected rational space (see Scheerer and Stelzer [30], but see also [7, Theorem 5.49]). A simple adaptation of the proof of [7, Theorem 5.49] yields the following generalization to maps; we omit the proof.

Proposition 1.7 If $f: X \rightarrow Y$ is a map between simply-connected rational spaces, then $\operatorname{Qcat}(f)=\operatorname{Mcat}(f)$.

\section{Categorical sequences}

In this section we will define our object of study, the categorical sequence associated to a space $X$. To ensure that our sequences are well-defined, we must first prove some results concerning the relative category of an $n$-skeleton.

\subsection{Relative category of skeleta}

Since we usually think of an $n$-skeleton as a subspace of $X$, we will sometimes write cat $_{X}\left(X_{n}\right)$ instead of $\operatorname{cat}(i)$ when $i: X_{n} \rightarrow X$ is an $n$-skeleton.

Proposition 2.1 For fixed $n$, the integer cat ${ }_{X}\left(X_{n}\right)$ depends only on the homotopy type of $X$, and not on the choice of $n$-skeleton.

Proof Let $i: A \rightarrow X$ and $j: B \rightarrow X$ be two $n$-skeleta of $X$ and consider the diagram

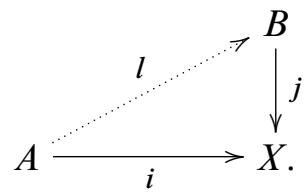

Algebraic 83 Geometric Topology, Volume 6 (2006) 
Since $j$ is an $n$-equivalence and $A$ is $n$-dimensional, there is a lift $l: A \rightarrow B$ such that $j \circ l \simeq i$ (see Switzer [35, Theorem 6.31]). It follows that $\operatorname{cat}_{X}(A)=\operatorname{cat}(i) \leq \operatorname{cat}(j)$ (see Berstein and Ganea $[2,1.4]$ ). Since the situation is symmetrical, we also have $\operatorname{cat}(j) \leq \operatorname{cat}(i)$.

It can be conceptually easier to work with the Lusternik-Schnirelmann category of spaces rather than of maps. Happily, there is no difference between the two for skeleta.

Proposition 2.2 If $X$ is $(c-1)$-connected and $i: X_{n} \rightarrow X$ is an $n$-skeleton with $n \geq c$, then

(a) $\operatorname{cat}\left(X_{n}\right)=\operatorname{cat}(i)$,

(b) $\operatorname{Qcat}\left(X_{n}\right)=\operatorname{Qcat}(i)$, and

(c) if $X$ is a rational space and $i: X_{n} \rightarrow X$ is a rational $n$-skeleton for $X$, then $\operatorname{cat}\left(X_{n}\right)=\operatorname{Mcat}(i)$.

Proof We begin by proving (a). It is trivial that $\operatorname{cat}_{X}\left(X_{n}\right) \leq \operatorname{cat}\left(X_{n}\right)$; we wish to prove the reverse inequality. Assume that $\operatorname{cat}_{X}\left(X_{n}\right)=k$; we will show that cat $\left(X_{n}\right) \leq k$. Since $n \geq c$, the map $i_{*}: \pi_{c}\left(X_{n}\right) \rightarrow \pi_{c}(X)$ is nontrivial, and hence $k \geq 1$. Now consider the diagram

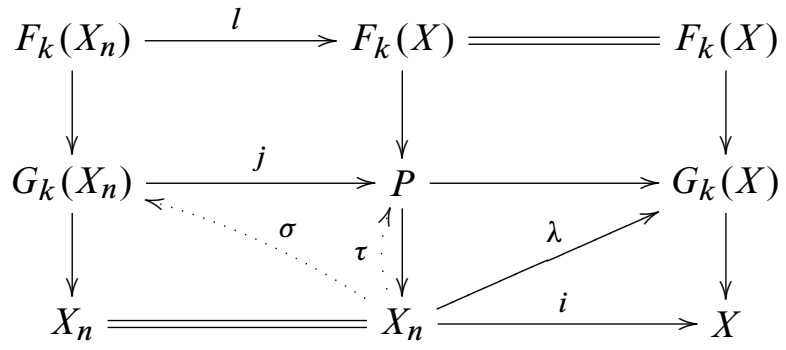

in which the bottom right square is a pullback. Since cat ${ }_{X}\left(X_{n}\right)=k$ there is a lift $\lambda$ of $i$. By the pullback property, there is a section $\tau: X_{n} \rightarrow P$.

According to [7, Lemma 6.26], the map $l: F_{k}\left(X_{n}\right) \rightarrow F_{k}(X)$ is an $(n+k c-1)-$ equivalence since $k \geq 1$, and it follows that $j$ is also a $(n+k c-1)$-equivalence. Since $n \leq n+k c-1$, it follows that there is a (unique) map $\sigma: X_{n} \rightarrow G_{k}\left(X_{n}\right)$ with $j \circ \sigma=\tau$ [35, Theorem 6.31]. This $\sigma$ is a section (up to homotopy) of the fibration $G_{k}\left(X_{n}\right) \rightarrow X_{n}$, and so $\operatorname{cat}\left(X_{n}\right) \leq k$.

The key to the proof of part (a) is the fact that $l: F_{k}\left(X_{n}\right) \rightarrow F_{k}(X)$ is an $(n+k c-1)-$ equivalence. But this implies that $Q l: Q F_{k}\left(X_{n}\right) \rightarrow Q F_{k}(X)$ is also an $(n+k c-1)-$ equivalence, and so the proof of (a) can be used again to show Qcat $(i)=\operatorname{Qcat}\left(X_{n}\right)$. 
It remains to prove (c). For this we simply compute

$$
\begin{aligned}
\operatorname{cat}\left(X_{n}\right) & =\operatorname{Mcat}\left(X_{n}\right) & & \text { by Hess [21, Theorem 0] } \\
& =\operatorname{Qcat}\left(X_{n}\right) & & \text { by Scheerer and Stelzer [30] } \\
& =\operatorname{Qcat}(i) & & \text { by part (b) } \\
& =\operatorname{Mcat}(i) & & \text { by Proposition 1.7. }
\end{aligned}
$$

This completes the proof.

Remark 2.3 The proof of Proposition 2.2(a) is an adaptation of the proof of [12, Theorem 1]. The argument actually works equally well with $i: X_{n} \rightarrow X$ replaced by any $n$-equivalence $f: Z \rightarrow X$ with $\operatorname{dim}(Z) \leq n+k c-1$. The conclusion in this case is that $\operatorname{cat}(f)=\operatorname{cat}(Z)=k$.

\subsection{Sequences from topology and algebra}

We will be concerned with sequences whose values are either nonnegative integers or $\infty$; thus a sequence is a function $\sigma: \mathbb{N} \rightarrow \mathbb{N} \cup\{\infty\}$. We say that $\sigma \leq \tau$ if $\sigma(k) \leq \tau(k)$ for each $k \geq 0$. We write $\sigma<\tau$ if $\sigma \leq \tau$ and $\sigma \neq \tau(\sigma<\tau$ does not mean that $\sigma(k)<\tau(k)$ for every $k$ ). If $\sigma$ is increasing, then the length of $\sigma$ is $\sup \{k \mid \sigma(k)<\infty\}$.

In view of Propositions 2.1 and 2.2, we may make the following definition.

Definition 2.4 The categorical sequence of a CW complex $X$ is the sequence $\sigma_{X}: \mathbb{N} \rightarrow \mathbb{N} \cup\{\infty\}$ defined by

$$
\sigma_{X}(k)=\inf \left\{n \mid \operatorname{cat}_{X}\left(X_{n}\right) \geq k\right\}
$$

Remark 2.5 The following elementary observations about categorical sequences will be used frequently in what follows.

(a) $\sigma_{X}$ is an invariant of the weak homotopy type of $X$.

(b) If $X$ is $(c-1)$-connected but not $c$-connected, then $\sigma_{X}(0)=0$ and $\sigma_{X}(1)=c$.

(c) The finite values of $\sigma_{X}$ are strictly increasing.

(d) If $\sigma_{X}(k)=n$, then $X_{n} \neq X_{n-1}$ in every cellular decomposition of $X$. In particular, if $X$ is simply-connected and $\sigma_{X}(k)=n$, then $H^{n}(X) \neq 0$ for some coefficients (see Theorem 3.4(b) below).

(e) If $X$ is finite-dimensional, then $\operatorname{cat}(X)=$ length $\left(\sigma_{X}\right)$; if $X$ is infinite-dimensional, then length $\left(\sigma_{X}\right) \leq \operatorname{cat}(X) \leq 2 \cdot$ length $\left(\sigma_{X}\right)$ (see Hardie [20]).

(f) In particular, cat $(X)=\infty$ if and only if length $\left(\sigma_{X}\right)=\infty$. 
(g) If $\sigma_{X} \leq \sigma_{Y}$ and $Y$ is finite-dimensional, then $\operatorname{cat}(X) \geq \operatorname{cat}(Y)$.

Before proceeding further, we give some examples.

Example 2.6 (a) As is well-known, the integral cohomology of the symplectic group $\operatorname{Sp}(2)$ is $H^{*}(\operatorname{Sp}(2))=\Lambda\left(x_{3}, x_{7}\right)$, an exterior algebra on generators in dimensions 3 and 7. It follows from Theorem 3.4(b) that the only possible finite values for $\sigma_{X}(k)$ are 0,3,7 and 10. Since it is known (see Schweitzer [31, Example 4.4]) that $\operatorname{cat}(S p(2))=3, \sigma_{S p(2)}(3)<\infty$, and hence

$$
\sigma_{S p(2)}=(0,3,7,10, \infty, \infty, \ldots) .
$$

(b) Define a Sullivan algebra $\mathcal{M}=\Lambda\left(x_{3}, y_{3}, z_{5}\right)$ with $d\left(z_{5}\right)=x_{3} y_{3}$, and let $X$ be a rational space whose minimal model is isomorphic to $\mathcal{M}$ (this algebra and space appear in [11, page 387]). The nontrivial cohomology of $X$ is

$$
\begin{aligned}
H^{3}(X) & =\mathbb{Q}[x] \oplus \mathbb{Q}[y] \\
H^{8}(X) & =\mathbb{Q}[x z] \oplus \mathbb{Q}[y z] \\
H^{11}(X) & =\mathbb{Q}[x y z]
\end{aligned}
$$

where brackets indicate cohomology classes. Thus cat $(X) \leq 3$, and since cat $(X) \geq$ $\operatorname{wgt}([x y z])=3$, we have $\operatorname{cat}(X)=3$. This forces $\sigma_{X}=(0,3,8,11, \infty, \infty, \ldots)$.

(c) The 'finite-dimensional' hypothesis in Remark 2.5(g) cannot be removed. Roitberg has shown that the cofibers $C$ of certain (phantom) maps $f: \Sigma K(\mathbb{Z}, 5) \rightarrow S^{4}$ have the property that $C_{n}$ is a suspension for all $n$, but cat $(C)=2$. Thus $\sigma_{C}=$ $(0,4, \infty, \infty, \ldots)=\sigma_{S^{4}}$, but $\operatorname{cat}(C)=2>1=\operatorname{cat}\left(S^{4}\right)$.

We will often abbreviate a sequence by deleting any terms known to be infinite. Thus, for example, we could summarize the results of Example 2.6(a,b) by writing $\sigma_{S p(2)}=$ $(0,3,7,10)$ and $\sigma_{X}=(0,3,8,11)$. If we were unsure of the later values of the sequence, we would write, for example, $\sigma_{S p(2)}=(0,3,7, \ldots)$; knowing that cat $(S p(2)) \leq 3$, we might write $\sigma_{S p(2)}=(0,3,7, a)$, where $a=10$ or $a=\infty$.

We will also make use of the algebraic product length sequence of a nonnegatively graded augmented CGA $\mathcal{A}$, defined by

$$
\sigma_{\mathcal{A}}(k)=\inf \left\{n \mid \exists \text { nontrivial } k \text {-fold products in } \mathcal{A}^{n}\right\} .
$$

If each of $P$ and $Q$ is either a space or a graded algebra, then it may happen that $\sigma_{P}=\sigma_{Q}$. If so, then we say that $P$ and $Q$ are isosequential. 
Example 2.7 (a) The spaces

$$
\overbrace{S^{2} \times \cdots \times S^{2}}^{n \text { factors }} \text { and } \mathbb{C P}^{n}
$$

are easily seen to be isosequential.

(b) It is easy to verify that $\sigma_{\mathbb{C P} \infty}=(0,2,4,6, \ldots)$; it is even easier to check that if $\mathcal{A}=H^{*}\left(\mathbb{C P}^{\infty} ; \mathbb{Q}\right), \sigma_{\mathcal{A}}=(0,2,4,6, \ldots)$. Thus the space $\mathbb{C P}^{\infty}$ and the graded algebra $H^{*}\left(\mathbb{C P}^{\infty} ; \mathbb{Q}\right)$ are isosequential.

(c) Let $X$ be the space of Example 2.6(b). Then $\sigma_{X}=(0,3,8,11)$, but $\sigma_{H^{*}(X)}=$ $(0,3,11)$, and $\sigma_{\mathcal{M}(X)}=(0,3,6,11)$, so $X$ is not isosequential with either $H^{*}(X)$ or $\mathcal{M}(X)$. Instead, these sequences are related by the string of strict inequalities $\sigma_{\mathcal{M}(X)}<\sigma_{X}<\sigma_{H^{*}(X)}$.

\section{Inequalities between sequences}

One of our goals is to develop techniques for computing categorical sequences $\sigma_{X}$. As with formulas for the calculation of $\operatorname{cat}(X)$, many of our results for sequences come in the form of inequalities.

\subsection{Inequalities for general spaces}

We begin by dispensing with wedges and retracts.

Proposition 3.1 Let $X$ and $Y$ be any two spaces. Then

(a) $\sigma_{X \vee Y}(k)=\min \left\{\sigma_{X}(k), \sigma_{Y}(k)\right\}$, and

(b) if $X$ is a homotopy retract of $Y$, then $\sigma_{X} \geq \sigma_{Y}$.

Proof Part (a) follows from the formula cat $(f \vee g)=\max \{\operatorname{cat}(f)$, $\operatorname{cat}(g)\}$. For (b), we consider the homotopy commutative diagram

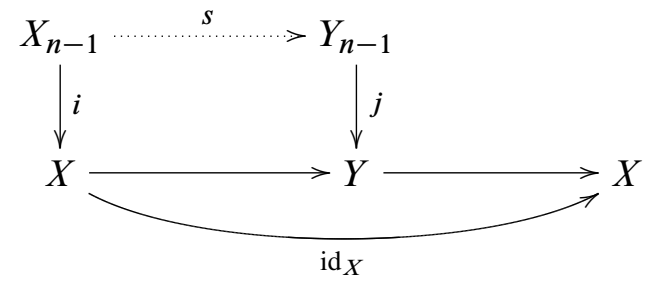

in which the map $s$ exists by cellular approximation. It follows from the commutativity of the diagram that $\operatorname{cat}_{X}\left(X_{n-1}\right)=\operatorname{cat}(i) \leq \operatorname{cat}(j)=\operatorname{cat}_{Y}\left(Y_{n-1}\right)$. Now $\sigma_{Y}(k)=n$ 
implies that $\operatorname{cat}_{Y}\left(Y_{n-1}\right)<k$ and hence that $\operatorname{cat}_{X}\left(X_{n-1}\right)<k$. Therefore $\sigma_{X}(k) \geq n=$ $\sigma_{Y}(k)$.

Our next result recasts the classical cup length lower bound for Lusternik-Schnirelmann category in terms of sequences.

Proposition 3.2 For any space $X$ and any ring $R, \sigma_{X} \leq \sigma_{H^{*}(X ; R)}$.

Proof Suppose that $\sigma_{H^{*}(X)}(k)=n$, so there is a nontrivial $k$-fold cup product $u_{1} \cdots u_{k} \in H^{n}(X)$. Let $i: X_{n} \rightarrow X$ be an $n$-skeleton. Then $i$ induces an injection $i^{*}: H^{n}(X) \rightarrow H^{n}\left(X_{n}\right)$, so $i^{*}\left(u_{1} \cdots u_{k}\right)=i^{*}\left(u_{1}\right) \cdots i^{*}\left(u_{k}\right) \neq 0 \in H^{n}\left(X_{n}\right)$. Therefore $\operatorname{cat}\left(X_{n}\right) \geq k$ (see Cornea, Lupton, Oprea and Tanré [7, Proposition 1.5]) and so $\sigma_{X}(k) \leq n$

Proposition 3.2 can be used to determine the categorical sequence of a product of spheres. This simple corollary will play an important role in our characterization of the categorical sequences of formal rational spaces (\$5).

Corollary 3.3 If $X=S^{n_{1}} \times \cdots \times S^{n_{r}}$ with $n_{1} \leq n_{2} \leq \cdots \leq n_{r}$, then $\sigma_{X}$ is given by the formula $\sigma_{X}(k)=\sigma_{H^{*}(X)}(k)=n_{1}+n_{2}+\cdots+n_{k}$ for $1 \leq k \leq r$ and $\sigma_{X}(k)=\infty$ for $k>r$.

Proof Clearly $\sigma_{H^{*}(X)}(k)=n_{1}+n_{2}+\cdots+n_{k}$, and Proposition 3.2 implies that $\sigma_{X} \leq \sigma_{H^{*}(X)}$. For the reverse inequality, let

$$
X(k)=\left\{\left(x_{1}, \ldots, x_{r}\right) \mid \text { at least } r-k \text { entries are } *\right\} \subseteq X .
$$

It is well-known that $X(0), X(1), \ldots, X(r)$ constitute a (spherical) cone decomposition of $X$. Furthermore, $X(k-1)$ contains the cellular $\left(n_{1}+n_{2}+\cdots+n_{k}-1\right)$-skeleton of $X$, and so

$$
\operatorname{cat}\left(X_{n_{1}+n_{2}+\cdots+n_{k}-1}\right) \leq \operatorname{cat}(X(k-1))<k .
$$

Therefore $\sigma_{X}(k) \geq n_{1}+n_{2}+\cdots+n_{k}=\sigma_{H^{*}(X)}(k)$.

The following theorem gives surprisingly strong algebraic control over categorical sequences. The proofs of parts (b) and (c) in full generality depend on the positive solution to Whitehead's Problem; but they are valid in ordinary ZFC set theory if $X$ is of finite type.

Theorem 3.4 For any space $X$,

(a) $\sigma_{X}(k+l) \geq \sigma_{X}(k)+\sigma_{X}(l)$, 
(b) if $X$ is simply-connected and $\sigma_{X}(k)=n$, then $H^{n}(X ; A) \neq 0$ for some coefficient group $A$, and

(c) if equality occurs in (a) and $X$ is simply-connected, then the cup product

$$
H^{k}(X ; A) \otimes H^{l}(X ; B) \rightarrow H^{k+l}(X ; A \otimes B)
$$

is nontrivial for some choice of coefficients.

Proof Write $\sigma_{X}(k)=a$ and $\sigma_{X}(l)=b$. Then $\operatorname{cat}\left(X_{a-1}\right)=k-1$ and $\operatorname{cat}\left(X_{b-1}\right)=$ $l-1$, which means that there are factorizations

$$
(X, *) \rightarrow\left(X, X_{a-1}\right) \rightarrow(X, *)^{k} \quad \text { and } \quad(X, *) \rightarrow\left(X, X_{b-1}\right) \rightarrow(X, *)^{l}
$$

of $\Delta_{k}$ and $\Delta_{l}$, up to homotopy of pairs. Putting these together using cellular approximation and the factorization $\Delta_{k+l}=\left(\Delta_{k} \times \Delta_{l}\right) \circ \Delta_{2}$, we obtain the homotopy-commutative diagram of pairs

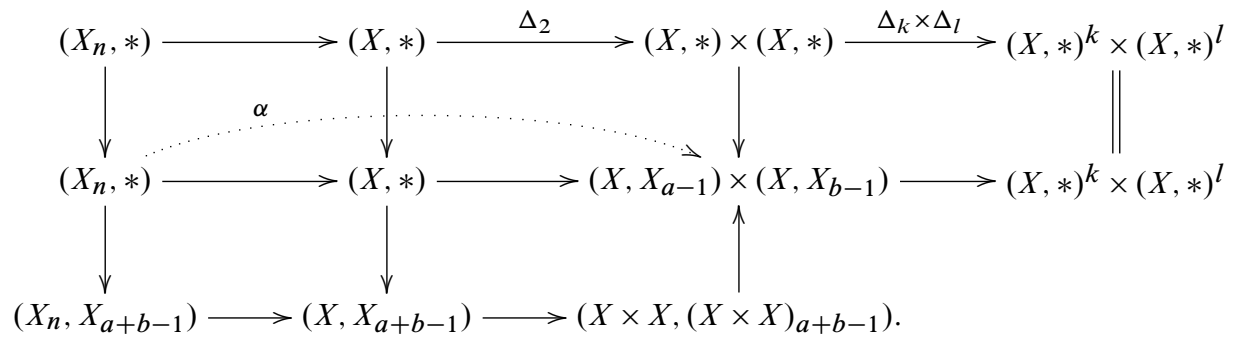

Taking $n=a+b-1$ we see that $\left.\Delta_{k+l}\right|_{X_{a+b-1}}$ factors, up to homotopy of pairs, through $\left(X_{a+b-1}, X_{a+b-1}\right)$, and so cat $X_{X}\left(X_{a+b-1}\right)<k+l$ by the Whitehead definition and Proposition 2.2. Therefore $\sigma_{X}(k+l) \geq a+b$, proving (a).

Now we prove part (b). If $\sigma_{X}(k)=n$, then cat $X\left(X_{n}\right)>$ cat $_{X}\left(X_{n-1}\right)$, so $X$ does not have an $(n-1)$-dimensional $n$-skeleton. By Lemma 1.3(c), then, it cannot be that $H^{n}(X ; A)=0$ for all $A$.

To prove the statement (c) about cup products, we first recall that by Theorem 3.4(b), if $\sigma_{X}(i)=m$, then $H^{m}(X ; A) \neq 0$ for some coefficient group $A$. Let $u \in H^{m}(X ; A)$ be nonzero, and interpret it as a map $u: X \rightarrow K(A, m)$. This map factors

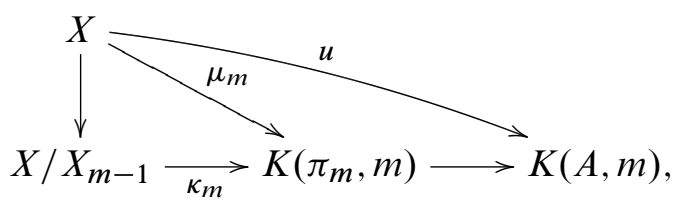

where $\pi_{m}=\pi_{m}\left(X / X_{m-1}\right)$. Since $u \not z *, \mu_{m} \not z *$ as well. Note also that $K\left(\pi_{m}, m\right)$ may be constructed from $X / X_{m-1}$ by attaching cells of dimension $m+2$ and higher, so $\kappa_{m}$ is an $(m+1)$-equivalence. 
Since $\left(X, X_{a-1}\right) \times\left(X, X_{b-1}\right)=\left(X \times X, X \times X_{b-1} \cup X_{a-1} \times X\right)$ is an $(a+b-1)-$ connected pair and $X \times X_{b-1} \cup X_{a-1} \times X$ is 1-connected, we apply the Blakers-Massey theorem (see Switzer [35, Corollary 6.22]) to conclude that the collapse map

$$
\left(X, X_{a-1}\right) \times\left(X, X_{b-1}\right) \rightarrow\left(X / X_{a-1} \wedge X / X_{b-1}, *\right)
$$

is an $(a+b+1)$-equivalence.

Assuming $\sigma_{X}(k+l)=\sigma_{X}(k)+\sigma_{X}(l)=a+b$, we may set $n=a+b$ in the diagram of part (a) and conclude that the composite map $\left(X_{a+b}, *\right) \rightarrow\left(X, X_{a-1}\right) \times\left(X, X_{b-1}\right)$ is nontrivial. Because the collapse map is an $(a+b+1)$-equivalence and $X_{a+b}$ is $(a+b)$-dimensional, we see that the composition

$$
\left(X_{a+b}, *\right) \rightarrow\left(X, X_{a-1}\right) \times\left(X, X_{b-1}\right) \rightarrow\left(X / X_{a-1} \wedge X / X_{b-1}, *\right)
$$

is also nontrivial. Now the desired cup product is

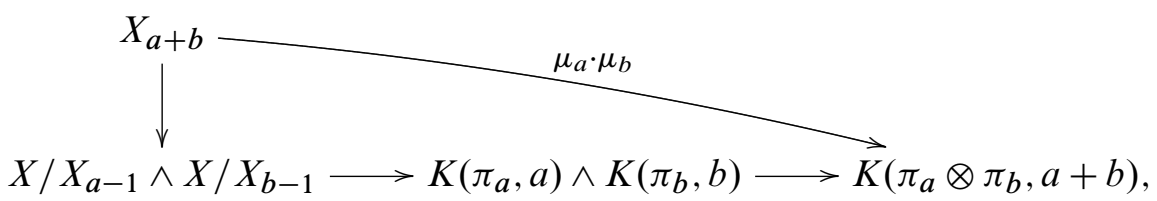

and it is nontrivial because the horizontal maps are all $(a+b+1)$-equivalences and $X_{a+b}$ is $(a+b)$-dimensional.

The following elementary computation illustrates the use of Theorem 3.4.

Example 3.5 Let us consider the exceptional Lie group $G_{2}$. It is known (see Mimura and Toda [25]) that $H^{*}\left(G_{2} ; \mathbb{Z} / 2\right) \cong\left(\mathbb{Z} / 2\left[x_{3}\right] /\left(x_{3}^{4}\right)\right) \otimes \Lambda\left(x_{5}\right)$. Therefore

$$
\sigma_{G_{2}} \leq \sigma_{H^{*}\left(G_{2} ; \mathbb{Z} / 2\right)}=(0,3,6,9,14, \infty, \ldots)
$$

by Proposition 3.2. On the other hand, we know $\sigma_{G_{2}}(1)=3$ by Remark $2.5(\mathrm{~b})$, so $\sigma_{G_{2}} \geq(0,3,6,9,12, \infty, \ldots)$ by Theorem $3.4(\mathrm{~b}, \mathrm{c})$; this determines $\sigma_{G_{2}}$ except for $\sigma_{G_{2}}(4)$. However, $H^{*}\left(G_{2} ; A\right)=0$ for $*=12,13$ and any abelian group $A$, so $\sigma_{G_{2}}(4) \neq 12,13$ by Theorem 3.4(b). We conclude that $\sigma_{G_{2}}=(0,3,6,9,14)$.

Theorem 3.4 implies the well-known result:

$$
\operatorname{cat}(X) \leq \frac{\operatorname{dimension}(X)}{\text { connectivity }(X)}
$$

In [16], Ganea generalized this familiar upper bound to obtain an upper bound for the category of $X$ in terms of the set of dimensions in which $H^{*}(X)$ is nontrivial. We 
now prove a further generalization by a completely different method. For a space $X$, let

$$
h(X)=\left\{n \mid \widetilde{H}^{n}(X ; G) \neq 0 \text { for some } G\right\} .
$$

Corollary 3.6 Let $X$ be simply-connected and of finite type with $\sigma_{X}(k)=n$. If there are integers $0<a_{1}<a_{2}<\cdots<a_{l}$ such that

$$
h(X) \subseteq I_{1} \cup I_{2} \cup \cdots \cup I_{l}
$$

where $I_{j}=\left[a_{j}, a_{j}+(n-1)\right]$ (brackets denote closed intervals in $\left.\mathbb{R}\right)$, then cat $(X)<$ $k(l+1)$.

Proof Consider the integers $\sigma_{X}(k j), j=1,2, \ldots$. We show by induction that $\sigma_{X}(k j) \geq a_{j}$. If $\sigma_{X}(k j)=\infty$ we are done, so we assume that this value is finite, and hence is an element of $h(X)$ by Theorem 3.4(b). Since $n \in h(X), a_{1} \leq n \leq a_{1}+(n-1)$. Now assume that $\sigma_{X}(k(j-1)) \geq a_{j-1}$. By Theorem 3.4(a),

$$
\sigma_{X}(k j) \geq \sigma_{X}(k(j-1))+\sigma_{X}(k) \geq a_{j-1}+n,
$$

which implies that $\sigma_{X}(k j) \notin \bigcup_{t<j} I_{t}$ and forces $\sigma_{X}(k j) \in \bigcup_{t \geq j} I_{t} \subseteq\left[a_{j}, \infty\right)$.

In particular, $\sigma_{X}(k l) \geq a_{l}$, and so $\sigma_{X}(k(l+1))>\sigma_{X}(k l)+\sigma_{X}(k)=a_{l}+n$ by Theorem 3.4(a). Thus $\sigma_{X}(k(l+1)) \notin h(X)$, and so $\sigma_{X}(k(l+1))=\infty$. Therefore $\operatorname{cat}(X)<k(l+1)$ by Remark 2.5(e), since the hypotheses imply that $X$ is weakly equivalent to a finite dimensional $\mathrm{CW}$ complex.

Ganea's theorem is the special case $k=1$ when $X$ is $(n-1)$-connected. It should be noted, though, that Ganea's result applies for strong category (ie, cone length), where ours only applies for ordinary Lusternik-Schnirelmann category. It would be interesting to know whether our generalization holds with cone length in place of category.

\subsection{Rational spaces}

The categorical sequence $\sigma_{X}$ for a rational space $X$ can be easily bounded above in terms of any one of its models.

Proposition 3.7 For any simply-connected rational space $X$, and any model $\mathcal{A}$ for $X, \sigma_{X} \geq \sigma_{\mathcal{A}}$.

Proof Write $\sigma_{\mathcal{A}}(k)=n$ and let $\mathcal{B}$ be the quotient of $\mathcal{A}$ by the differential ideal consisting of all elements of dimension $n$ or greater. Then $\operatorname{nil}(\mathcal{B})<k$ and the quotient 
$q: \mathcal{A} \rightarrow \mathcal{B}$ induces an isomorphism on cohomology in dimensions $<n-1$ and an injection in dimension $n-1$.

Let $\mathcal{N}$ be the Sullivan minimal model for $\mathcal{B}$ and let $r: \mathcal{M}(X) \rightarrow \mathcal{N}$ cover the map $q$. Then $r$ has a spatial realization $i: Z \rightarrow X$ such that $q^{*}=i^{*}: H^{*}(X) \rightarrow H^{*}(Z)$ (see Félix, Halperin and Thomas [11, Chapter 17]). It follows that $i: Z \rightarrow X$ is a rational $(n-1)$-skeleton. Since $\mathcal{M}(Z) \sim \mathcal{B}$ by construction and $\operatorname{nil}(\mathcal{B})<k$, we conclude using Theorem 1.6 that $\operatorname{cat}(Z)<k$. It follows that $\sigma_{X}(k) \geq n$.

Example 3.8 Let $(\mathcal{A}, d)$ be the CDGA with generators $x_{n}, y_{m}$ and $w_{n+m-1}$ (subscripts indicate dimension; $2 \leq n \leq m$ ) subject to the relations $x^{2}=y^{2}=w^{2}=0$ and with differential determined by $d x=d y=0$ and $d w=x y$. This is not a Sullivan algebra, but it does have a Sullivan model, $\mathcal{M}$, and $\mathcal{M}$ has a spatial realization, $X$. Then $\mathcal{A}$ is a model for $X$, and according to Proposition 3.7,

$$
\sigma_{X} \geq \sigma_{\mathcal{A}}=(0, n, n+m, 2(n+m)-1, \infty, \infty, \ldots) .
$$

But we can say even more, because the nonzero cohomology of $X$ occurs in dimensions $n, m, 2 n+m-1, n+2 m-1$ and $2(n+m)-1$. Since $X$ is indistinguishable from $S^{n} \vee S^{m}$ through dimension $n+m$, we know that $\sigma_{X}(2)>n+m$, and therefore $\sigma_{X}(2) \geq 2 n+m-1$. Thus

$$
\sigma_{X} \geq(0, n, 2 n+m-1,2(n+m)-1, \infty, \infty, \ldots) .
$$

Since $\mathcal{A}$ is finite-dimensional, so is $H^{*}(X)$, and we conclude that cat $(X) \leq 3$.

\section{Sequences and fibrations}

In this section we study the relationship between the sequences $\sigma_{F}, \sigma_{E}$ and $\sigma_{B}$ when $F \rightarrow E \rightarrow B$ is a fibration sequence. Our general result is the key to a mapping theorem for categorical sequences of rational spaces.

\subsection{General spaces}

Our first result is proved by a slight generalization of the method Hardie used to prove the main result of [19].

Proposition 4.1 Consider the diagram

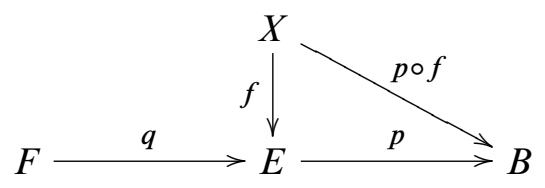

Algebraic 83 Geometric Topology, Volume 6 (2006) 
in which the bottom row is a fibration sequence. Then

$$
\operatorname{cat}(f)+1 \leq(\operatorname{cat}(p \circ f)+1) \cdot(\operatorname{cat}(q)+1) .
$$

Proof Suppose $\operatorname{cat}(p \circ f)=k$ and that $\operatorname{cat}(q)=l$. Then $X$ has a cover $X=$ $A_{0} \cup A_{1} \cup \cdots \cup A_{k}$ by subcomplexes such that $\left.(p \circ f)\right|_{A_{i}} \simeq *$ for each $i$. Since $p$ is a fibration with fiber $F,\left.f\right|_{A_{i}}$ factors (up to homotopy) as $j \circ g_{i}$, where $g_{i}: A_{i} \rightarrow F$. Therefore $\operatorname{cat}\left(\left.f\right|_{A_{i}}\right) \leq \operatorname{cat}(q)=l$ and so we can write $A_{i}=A_{i 0} \cup A_{i 1} \cup \ldots \cup A_{i l}$ where $\left.\left(q \circ g_{i}\right)\right|_{A_{i j}} \simeq *$. Thus $X=\bigcup_{i, j} A_{i j}$ where $0 \leq i \leq k$ and $0 \leq j \leq l$ and $\left.f\right|_{A_{i j}} \simeq *$ for all $i$ and $j$. Therefore $\operatorname{cat}(f)+1 \leq(k+1)(l+1)$.

Hardie's result is the special case in which $f=\mathrm{id}_{E}$. We are interested in the more general situation in which $f: X \rightarrow E$ is an $n$-skeleton.

Theorem 4.2 Let

$$
F \stackrel{q}{\longrightarrow} E \stackrel{p}{\longrightarrow} B
$$

be a fibration sequence and write $a=\operatorname{cat}(q) \leq \operatorname{cat}(F)$ and $b=\operatorname{cat}(p) \leq \operatorname{cat}(B)$. Then

(a) $\sigma_{E}(k(a+1)) \geq \sigma_{B}(k)$, and

(b) $\sigma_{E}(k(b+1)) \geq \sigma_{F}(k)$.

Proof Let $\sigma_{B}(k)=n$. Thus cat $\left(B_{n-1}\right)<k$ and we have to show that $\operatorname{cat}_{E}\left(E_{n-1}\right)<$ $k(a+1)$. Consider the homotopy-commutative diagram

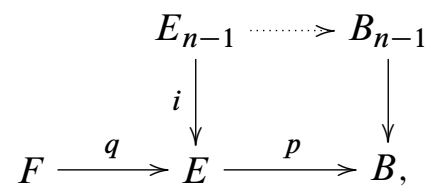

in which the dotted arrow exists by cellular approximation. According to Proposition 4.1,

$$
\begin{aligned}
\operatorname{cat}(i) & \leq(\operatorname{cat}(p \circ i)+1) \cdot(\operatorname{cat}(q)+1)-1 \\
& <\left(\operatorname{cat}\left(B_{n-1}\right)+1\right) \cdot(a+1) \\
& <k(a+1),
\end{aligned}
$$

proving (a).

For part (b), we let $\sigma_{F}(k)=n$, so $\operatorname{cat}\left(F_{n-1}\right)=k-1$. Choose an $(n-1)$-skeleton $i: E_{n-1} \rightarrow E$. Since cat $(p \circ i) \leq \operatorname{cat}(p)=b$, we can write $E_{n-1}=A_{0} \cup A_{1} \cup \cdots \cup A_{b}$ 
where $A_{j}$ is a subcomplex of $E_{n-1}$ (so $\left.\operatorname{dim}\left(A_{j}\right)<n\right)$ and $\left.(p \circ i)\right|_{A_{j}} \simeq *$ for each $j$. Thus $\left.i\right|_{A_{j}}$ factors (up to homotopy) through the $F \rightarrow E$, and so we have the diagram

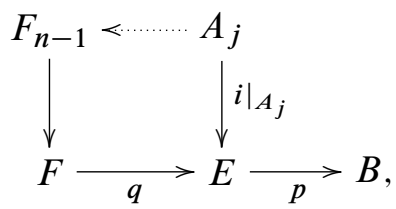

in which the dotted arrow exists by cellular approximation. This proves that cat $\left(\left.i\right|_{A_{j}}\right) \leq$ $\operatorname{cat}\left(F_{n-1}\right)=k-1$, and so cat $(i)<(b+1) k$, which implies the desired inequality $\sigma_{E}((b+1) k) \geq n$.

Remark 4.3 These inequalities are not the best possible. A quick look at the proof of Theorem 4.2 shows that, in studying the category of $E_{n}$, for example, the estimate $\operatorname{cat}(p \circ i) \leq b$ can be improved to $\operatorname{cat}(p \circ i) \leq \operatorname{cat}\left(B_{n}\right)$, and similarly for the second formula. We leave the cumbersome formulation of the sharper results to the reader.

Since the reverse formulas expressing $\sigma_{E}$ in terms of $\sigma_{B}$ and $\sigma_{F}$ are not entirely obvious, we record them here.

Corollary 4.4 In the situation of Theorem 4.2,

(a) $\sigma_{E}(k) \leq \sigma_{B}\left(\left\lceil\frac{k-a}{a+1}\right\rceil\right)$, and

(b) $\sigma_{E}(k) \leq \sigma_{F}\left(\left\lceil\frac{k-b}{b+1}\right\rceil\right)$.

In [8], Fadell and Husseini studied the Lusternik-Schnirelmann category of free loop spaces using a general result that relates the category of the fiber and the total space in a fibration sequence with a section. This result generalizes to a statement about categorical sequences.

\section{Corollary 4.5 Let}

$$
F \longrightarrow E \stackrel{p}{\longrightarrow} B
$$

be a fibration sequence. If $\Omega p$ has a section $s$, then $\sigma_{E} \leq \sigma_{F}$.

Proof Extend the given fibration sequence to the left to obtain

$$
\Omega E \underset{\Omega p}{\stackrel{s}{\rightleftarrows}} \Omega B \underset{\partial}{\longrightarrow} F \longrightarrow E .
$$

Since $\Omega p$ has a section, the map $\partial: \Omega B \rightarrow F$ is trivial. Thus cat $(\partial)=0$, and Theorem 4.2(a) implies $\sigma_{F}(k)=\sigma_{F}((\operatorname{cat}(\partial)+1) k) \geq \sigma_{E}(k)$. 
We can now expand upon the main homotopy-theoretical result of [8].

Example 4.6 Let $L(X)=\operatorname{map}\left(S^{1}, X\right)$ denote the free loop space on $X$. Evaluation at the basepoint determines a fibration $p: L(X) \rightarrow X$ with fiber $\Omega X$, and the map $s: x \mapsto l_{x}$, where $l_{x}$ is the constant map $l_{x}\left(S^{1}\right)=x$, is a section of $p$; thus $\Omega s$ is a section of $\Omega p$. Therefore Corollary 4.5 shows that

$$
\sigma_{L(X)} \leq \sigma_{\Omega X} .
$$

In particular, $\operatorname{cat}(L(X))=\infty$ if $\operatorname{cat}(\Omega X)=\infty$.

\subsection{A mapping theorem for sequences}

One of the most powerful early results concerning the Lusternik-Schnirelmann category of rational spaces is the Mapping Theorem (see Félix and Halperin [10]); the nice 'book proof' of this result (see Félix and Lemaire [13]) uses Proposition 4.1 in the special case $\operatorname{cat}(j)=0$. We use exactly the same argument to get an inequality for categorical sequences.

Proposition 4.7 Let $f: X \rightarrow Y$ be a map between rational spaces which induces an injective map $f_{*}: \pi_{*}(X) \rightarrow \pi_{*}(Y)$. Then $\sigma_{X} \geq \sigma_{Y}$.

Proof Let $q: F \rightarrow X$ be the homotopy fiber of $f$. According to the proof of the standard Mapping Theorem, the injectivity hypothesis on $f_{*}$ implies that $q \simeq *$ and so $\operatorname{cat}(q)=0$ [7, Theorem 4.11]. It now follows from Theorem 4.2 that $\sigma_{X}(k) \geq \sigma_{Y}(k)$ for all $k$.

\section{Formal sequences}

A simply-connected space $X$ is formal if its cohomology algebra, with trivial differential, is a model for $X$ [11, page 156]. In this section we characterize the categorical sequences of formal rational spaces in several ways.

First we show that formal rational spaces and their cohomology algebras are isosequential.

Proposition 5.1 If $X$ is a simply-connected formal rational space, then $\sigma_{X}=\sigma_{H^{*}(X)}$.

Proof By assumption, $H^{*}(X)$ is a model for $X$. Propositions 3.7 and 3.2 show that $\sigma_{H^{*}(X)} \leq \sigma_{X} \leq \sigma_{H^{*}(X)}$, which proves the result. 
Our main result in this section completely characterizes the sequences which can occur as categorical sequences of simply-connected rational formal spaces.

Theorem 5.2 The following conditions on a sequence $\sigma$ with $\sigma(1)>1$ are equivalent:

(a) $\sigma=\sigma_{\mathcal{A}}$ for some $C G A \mathcal{A}$,

(b) $\sigma(k+1) \geq \frac{k+1}{k} \sigma(k)$ for each $k$,

(c) $\sigma=\sigma_{W}$ where $W=\bigvee P_{i}$ and $P_{i}=\prod S^{n_{j}}$ is a product of spheres, and

(d) $\sigma=\sigma_{X}$ for some formal space $X$.

Before proceeding to the proof of Theorem 5.2 we need to establish a technical result about sequences. Let $0<k \leq n$ be integers, write $n=k x+r$ with $0 \leq r<k$ and let $r+s=k$. Define $\tau$ to be the sequence whose finite values are

$$
\tau=(0, x, 2 x, \ldots, s x, s x+(x+1), s x+2(x+1), \ldots, \underbrace{s x+r(x+1)}_{n}) .
$$

We call $\tau$ the optimal $k$-term sequence with $\tau(k)=n$.

Lemma 5.3 Assume that $\sigma$ is a sequence satisfying condition (b) of Theorem 5.2, and that $\sigma(k)<\infty$. Let $\tau$ be the optimal $k$-term sequence with $\tau(k)=\sigma(k)$. Then $\sigma \leq \tau$.

Proof This is clearly true for $j>k$, because $\tau(j)=\infty$ for such $j$. If $\sigma(j)>\tau(j)$ for some $j \leq k$, then $\sigma(j) \geq \tau(j)+1$, and so

$$
\sigma(j+1) \geq \frac{1}{j} \sigma(j)+\sigma(j) \geq \frac{1}{j} \sigma(j)+(\tau(j)+1)
$$

Now $\sigma(j)>\tau(j) \geq j x$, so $\frac{1}{j} \sigma(j)>x$. Therefore

$$
\sigma(j+1)>\tau(j)+(x+1) \geq \tau(j+1) .
$$

Inductively, we see that $\sigma(l)>\tau(l)$ for all $i \leq l \leq k$, which contradicts the hypothesis $\sigma(k)=\tau(k)$.

Proof of Theorem 5.2 We begin by proving that (a) implies (b). Let $\mathcal{A}$ be a CGA such that $\sigma=\sigma_{\mathcal{A}}$. If $\sigma=(0, n)$ has length 1 , then there is nothing to prove, so we proceed by induction, assuming that the implication is valid for sequences of length $\leq k$. Write $n=\sigma(k+1)=\sigma_{\mathcal{A}}(k+1)$. Then there is a nontrivial product $x_{1} x_{2} \cdots x_{k+1} \in \mathcal{A}^{n}$, where we write the terms in order so that $\left|x_{1}\right| \leq\left|x_{2}\right| \leq \cdots \leq\left|x_{k+1}\right|$. For $j \leq k+1$ we have

$$
x_{1} x_{2} \cdots x_{j} \neq 0 \in \mathcal{A}^{\left|x_{1}\right|+\left|x_{2}\right|+\cdots+\left|x_{j}\right|},
$$


so $\sigma_{\mathcal{A}}(j) \leq\left|x_{1}\right|+\left|x_{2}\right|+\cdots+\left|x_{j}\right|$ for each $j$. Since $\sigma_{\mathcal{A}}(k+1)=\left|x_{1}\right|+\left|x_{2}\right|+\cdots+$ $\left|x_{k+1}\right|$ by construction, we have

$$
\begin{aligned}
\sigma_{\mathcal{A}}(k+1)-\sigma_{\mathcal{A}}(k) & \geq\left(\left|x_{1}\right|+\cdots+\left|x_{k+1}\right|\right)-\left(\left|x_{1}\right|+\cdots+\left|x_{k}\right|\right) \\
& =\left|x_{k+1}\right| \\
& =\frac{1}{k} \overbrace{\left(\left|x_{k+1}\right|+\left|x_{k+1}\right|+\cdots+\left|x_{k+1}\right|\right)}^{k \text { terms }} \\
& \geq \frac{1}{k}\left(\left|x_{1}\right|+\left|x_{2}\right|+\cdots+\left|x_{k}\right|\right) \\
& \geq \frac{1}{k} \sigma_{\mathcal{A}}(k),
\end{aligned}
$$

which proves the result.

Next we prove that (b) implies (c) by induction on the length $k$ of the sequence $\sigma$. If $\sigma=(0, n)$, then $\sigma=\sigma_{S^{n}}$ and the result holds. Suppose now that the result is known for all sequences with length $\leq k$, and let $\sigma$ be a sequence with length $k+1$. Write $\bar{\sigma}$ for the sequence

$$
\bar{\sigma}(j)= \begin{cases}\sigma(j) & \text { if } j \leq k \\ \infty & \text { if } j>k\end{cases}
$$

Since length $(\bar{\sigma}) \leq k$, we can apply the inductive hypothesis, to find a wedge of products of spheres $W$ such that $\sigma_{W}=\bar{\sigma}$. Let $\tau$ be the optimal $(k+1)$-term sequence with $\tau(k+1)=\sigma(k+1)$, and define

$$
P=\overbrace{S^{x} \times S^{x} \times \cdots \times S^{x}}^{s \text { factors }} \times \overbrace{S^{x+1} \times S^{x+1} \times \cdots \times S^{x+1}}^{r \text { factors }} .
$$

Then $\sigma_{P}=\tau$ by Corollary 3.3, and Proposition 3.1 shows that

$$
\sigma_{W \vee P}(j)=\min \left\{\sigma_{W}(j), \sigma_{P}(j)\right\}
$$

for all $j$. For $j \leq k$, we have $\sigma_{W}(j)=\sigma(j) \leq \tau(j)=\sigma_{P}(j)$ by Lemma 5.3, so $\sigma_{W \vee P}=\sigma(j)$ for $j<k$ by Proposition 3.1(a). Also $\sigma_{P}(k+1)=\sigma(k+1)<\infty=$ $\sigma_{W}(k+1)$, so $\sigma_{W \vee P}(k+1)=\sigma(k+1)$.

The implication (c) $\Rightarrow$ (d) follows from the fact that the rationalization of a wedge of products of spheres is formal.

According to Proposition 5.1, if $X$ is a formal rational space, then $\sigma_{X}=\sigma_{H^{*}(X)}$. Thus (d) implies (a).

In view of Theorem 5.2, we define a formal sequence to be any sequence $\sigma$ which satisfies the condition

$$
\sigma(k+1) \geq \frac{k+1}{k} \sigma(k) \quad \text { for all } k .
$$


It is not true that every formal space is isosequential with its minimal model. For example, the minimal model of $S^{4}$ is $\Lambda\left(x_{4}, x_{7}\right)$, so

$$
\sigma_{S^{4}}=(0,4)>(0,4,8,12, \ldots)=\sigma_{\mathcal{M}\left(S^{4}\right)} .
$$

Our study of formal sequences grew out of a simple question: is every simply-connected rational space isosequential with a product of spheres, or a wedge of products of spheres, or a product of wedges of products of spheres, etc?

Any space constructed from spheres by repeatedly taking products and wedges is automatically formal [7, Example 5.4]. Using Theorem 5.2, we see that any such space is isosequential with a simple wedge of products of spheres. Furthermore, Theorem 5.2 reveals that our original question reduces to asking whether or not $\sigma_{X}$ is a formal sequence whenever $X$ is a rational space. We have already seen that this is not the case!

Example 5.4 The space $X$ of Example 2.6(b) is a rational space whose categorical sequence is $\sigma_{X}=(0,3,8,11)$. Since $11<\frac{3}{2} \cdot 8, \sigma_{X}$ is not a formal sequence. By Theorem 5.2, $X$ is not isosequential with any wedge of products of spheres.

\section{Products}

For two sequences $\sigma$ and $\tau$, we define a new sequence $\sigma * \tau$ by

$$
\sigma * \tau(k)=\min \{\sigma(i)+\sigma(j) \mid i+j=k\} .
$$

Our goal in this section is to prove a result linking the sequences $\sigma_{X \times Y}$ and $\sigma_{X} * \sigma_{Y}$. When the spaces in question are formal, this is not hard to do.

Proposition 6.1 Let $\mathcal{A}$ and $\mathcal{B}$ be simply-connected CGAs and let $X$ and $Y$ be simply-connected formal rational spaces. Then

(a) $\sigma_{\mathcal{A} \otimes \mathcal{B}}=\sigma_{\mathcal{A}} * \sigma_{\mathcal{B}}$, and

(b) $\sigma_{X \times Y}=\sigma_{X} * \sigma_{Y}$.

Proof We omit the easy proof of (a), and use it to prove (b) as follows: since $X, Y$ and $X \times Y$ are each formal and rational,

$$
\sigma_{X \times Y}=\sigma_{H^{*}(X \times Y)}=\sigma_{H^{*}(X) \otimes H^{*}(Y)}=\sigma_{H^{*}(X)} * \sigma_{H^{*}(Y)}=\sigma_{X} * \sigma_{Y}
$$

by Proposition 5.1 . 
The following conjecture seems quite plausible.

Conjecture A For simply-connected rational $X$ and $Y, \sigma_{X \times Y}=\sigma_{X} * \sigma_{Y}$.

Unfortunately, we have been unable to prove this. However, we can prove that there is an inequality relating these sequences.

Theorem 6.2 For simply-connected rational $X$ and $Y, \sigma_{X \times Y} \leq \sigma_{X} * \sigma_{Y}$.

Proof Let $\sigma_{X} * \sigma_{Y}(k)=n$. Thus there are $i$ and $j$ with $i+j=k, \sigma_{X}(i)=a$, $\sigma_{Y}(j)=b$, and $a+b=n$. Now let $i_{a} \times i_{b}: X_{a} \times Y_{b} \rightarrow X \times Y$ and compute

$$
\begin{aligned}
\operatorname{cat}\left((X \times Y)_{n}\right) & \geq \operatorname{cat}_{X \times Y}\left(X_{a} \times Y_{b}\right) & & \\
& \geq \operatorname{Mcat}\left(i_{a} \times i_{b}\right) & & \text { by Proposition 2.2(c) } \\
& =\operatorname{Mcat}\left(i_{a}\right)+\operatorname{Mcat}\left(i_{b}\right) & & \text { by Parent [26, Theorem 2] } \\
& =\operatorname{cat}\left(X_{a}\right)+\operatorname{cat}\left(X_{b}\right) & & \text { by Proposition 2.2(c) } \\
& =k, & &
\end{aligned}
$$

which means that $\sigma_{X \times Y}(k) \leq n=\sigma_{X} * \sigma_{Y}(k)$,

The inequality of Theorem 6.2 fails when the spaces are not rational, as the following example demonstrates.

Example 6.3 Iwase [22] has constructed a space $X=S^{2} \cup D^{10}$ with the property that $\operatorname{cat}\left(X \times S^{k}\right)=\operatorname{cat}(X)=2$ for all $k \geq 2$. The categorical sequences for $X$ and $S^{2}$ are $\sigma_{X}=(0,2,10, \infty, \ldots)$ and $\sigma_{S^{2}}=(0,2, \infty, \ldots)$, respectively. Now we have $\sigma_{X} * \sigma_{S^{2}}=(0,2,4,12, \infty, \ldots)<(0,2,4, \infty, \ldots)=\sigma_{X \times S^{2}}$.

Nevertheless, the following conjecture seems reasonable.

Conjecture B For general spaces $X$ and $Y, \sigma_{X \times Y} \geq \sigma_{X} * \sigma_{Y}$.

Conjecture B, together with Theorem 6.2, implies Conjecture A.

\section{The Mislin genus of $S p(3)$}

In this final section we use categorical sequences to give a simple proof of a theorem of Ghienne [18].

The Mislin genus of a nilpotent space $X$ is the set $\mathcal{G}(X)$ of homotopy types of nilpotent spaces $Y$ such that the $p$-localizations $X_{(p)}$ and $Y_{(p)}$ are homotopy equivalent for 
every prime $p$. McGibbon [24, Section 8] asked whether Lusternik-Schnirelmann category is an invariant of Mislin genus; that is, if $X \in \mathcal{G}(Y)$, does it follow that $\operatorname{cat}(X)=\operatorname{cat}(Y)$ ? This is known to be false for certain infinite-dimensional spaces (see Roitberg [28]), but the question remains open for finite complexes $Y$.

In [18], Ghienne proved that McGibbon's conjecture holds in the special case $Y=$ $S p(3)$. We use a sequence computation to give a simple alternative proof of this result.

Theorem 7.1 (Ghienne) If $X \in \mathcal{G}(S p(3))$, then $\operatorname{cat}(X)=5$.

Proof According to Fernández-Suárez, Gómez-Tato, Strom and Tanré [14], and Iwase and Mimura [23], wcat $(S p(3))=\operatorname{cat}(S p(3))=5$. Since weak category is a genus invariant, we have

$$
\operatorname{cat}(X) \geq \operatorname{wcat}(X)=\operatorname{wcat}(S p(3))=5
$$

for any space $X \in \mathcal{G}(S p(3))$. It remains to show that cat $(X) \leq 5$ for every $X \in$ $\mathcal{G}(S p(3))$. In fact, we prove the following stronger statement: any simply-connected space $X$ whose cohomology ring $H^{*}(X ; \mathbb{Z})$ is isomorphic to $H^{*}(\operatorname{Sp}(3) ; \mathbb{Z})$ must have $\operatorname{cat}(X) \leq 5$.

The categorical sequence $\sigma_{X}$ clearly has $\sigma_{X}(1)=3$ and $\sigma_{X}(2) \geq 7$ by Theorem 3.4(b). By Theorem 3.4(a), $\sigma_{X}(4) \geq \sigma_{X}(2)+\sigma_{X}(2) \geq 14$. Furthermore, $\sigma_{X}(4)>14$ by Theorem 3.4(c), because the cup product $H^{7}(X) \otimes H^{7}(X) \rightarrow H^{14}(X)$ is trivial. Now we have $\sigma_{X}(4) \geq 18$ by Theorem 3.4(b), and hence $\sigma_{X}(5) \geq \sigma_{X}(4)+\sigma_{X}(1)=21$. From this we immediately conclude that $\operatorname{cat}(X)=\operatorname{cat}\left(X_{21}\right) \leq 5$.

McGibbon's conjecture for finite complexes is equivalent to the following conjecture for finite type spaces.

Conjecture $\mathbf{C}$ If $X$ is a nilpotent space of finite type, then $\sigma_{Y}=\sigma_{X}$ for every $Y \in \mathcal{G}(X)$.

Conjecture $\mathrm{C}$ is easily seen to be valid for $X=S p(2)$. We can also verify the conjecture for $X=S p(3)$.

Corollary 7.2 If $X \in \mathcal{G}(S p(3))$, then $\sigma_{X}=\sigma_{S p(3)}=(0,3,7,10,18,21)$.

Proof The proof of Theorem 7.1 shows that if $H^{*}(X) \cong H^{*}(\operatorname{Sp}(3))$ then $\sigma_{X} \geq$ $(0,3,7,10,18,21)$. If $\operatorname{cat}(X)=5$, then $\sigma_{X}(5) \leq 21$, and this implies $\sigma_{X}(2)=7$. Now [34, Theorem 8] implies that $\operatorname{cat}\left(X_{10}\right)=3$, and hence $\sigma_{X}(3)=10$. The analysis used in the proof of Theorem 7.1 shows that $\sigma_{X}=(0,3,7,10,18,21)$. 


\section{References}

[1] A Ambrosetti, V Coti Zelati, Critical points with lack of compactness and singular dynamical systems, Ann. Mat. Pura Appl. (4) 149 (1987) 237-259 MR932787

[2] I Berstein, T Ganea, The category of a map and of a cohomology class, Fund. Math. $50(1961 / 1962) 265-279$ MR0139168

[3] I Berstein, P J Hilton, Category and generalized Hopf invariants, Illinois J. Math. 4 (1960) 437-451 MR0126276

[4] E H Brown, Jr, A H Copeland, Jr, An homology analogue of Postnikov systems, Michigan Math. J 6 (1959) 313-330 MR0110096

[5] G Cicortaş, Categorical sequences and applications, Studia Univ. Babeş-Bolyai Math. 47 (2002) 31-39 MR1989588

[6] G Cicortaş, Relatively and G-categorical sequences and applications, from: "Proceedings of "BOLYAI 200" International Conference on Geometry and Topology", Cluj Univ. Press, Cluj-Napoca (2003) 67-74 MR2112613

[7] O Cornea, G Lupton, J Oprea, D Tanré, Lusternik-Schnirelmann category, Mathematical Surveys and Monographs 103, American Mathematical Society, Providence, RI (2003) MR1990857

[8] E Fadell, S Husseini, A note on the category of the free loop space, Proc. Amer. Math. Soc. 107 (1989) 527-536 MR984789

[9] Y Félix, La dichotomie elliptique-hyperbolique en homotopie rationnelle, Astérisque (1989) 187 MR1035582

[10] Y Félix, S Halperin, Rational LS category and its applications, Trans. Amer. Math. Soc. 273 (1982) 1-38 MR664027

[11] Y Félix, S Halperin, J-C Thomas, Rational homotopy theory, Graduate Texts in Mathematics 205, Springer, New York (2001) MR1802847

[12] Y Felix, S Halperin, J-C Thomas, Lusternik-Schnirelmann category of skeleta, Topology Appl. 125 (2002) 357-361 MR1933583

[13] Y Félix, J-M Lemaire, On the mapping theorem for Lusternik-Schnirelmann category, Topology 24 (1985) 41-43 MR790674

[14] L Fernández-Suárez, A Gómez-Tato, J Strom, D Tanré, The LusternikSchnirelmann category of $\mathrm{Sp}(3)$, Proc. Amer. Math. Soc. 132 (2004) 587-595 MR2022385

[15] R H Fox, On the Lusternik-Schnirelmann category, Ann. of Math. (2) 42 (1941) 333-370 MR0004108

[16] T Ganea, Upper estimates for the Ljusternik-Snirel'man category, Soviet Math. Dokl. 2 (1961) 180-183 MR0132546 
[17] T Ganea, A generalization of the homology and homotopy suspension, Comment. Math. Helv. 39 (1965) 295-322 MR0179791

[18] P Ghienne, The Lusternik-Schnirelmann category of spaces in the Mislin genus of $\mathrm{Sp}$ (3), from: "Lusternik-Schnirelmann category and related topics (South Hadley, MA, 2001)", Contemp. Math. 316, Amer. Math. Soc., Providence, RI (2002) 121-126 MR1962158

[19] K A Hardie, A note on fibrations and category, Michigan Math. J. 17 (1970) 351-352 MR0283795

[20] KA Hardie, On the category of the double mapping cylinder, Tôhoku Math. J. (2) 25 (1973) 355-358 MR0370558

[21] K P Hess, A proof of Ganea's conjecture for rational spaces, Topology 30 (1991) 205-214 MR1098914

[22] N Iwase, Ganea's conjecture on Lusternik-Schnirelmann category, Bull. London Math. Soc. 30 (1998) 623-634 MR1642747

[23] N Iwase, M Mimura, L-S categories of simply-connected compact simple Lie groups of low rank, from: "Categorical decomposition techniques in algebraic topology (Isle of Skye, 2001)”, Progr. Math. 215, Birkhäuser, Basel (2004) 199-212 MR2039767

[24] C A McGibbon, The Mislin genus of a space, from: "The Hilton Symposium 1993 (Montreal, PQ)", CRM Proc. Lecture Notes 6, Amer. Math. Soc., Providence, RI (1994) 75-102 MR1290585

[25] M Mimura, H Toda, Topology of Lie groups. I, II, Translations of Mathematical Monographs 91, American Mathematical Society, Providence, RI (1991) MR1122592

[26] P-E Parent, LS category: product formulas, Topology Appl. 106 (2000) 35-47 MR1769330

[27] PH Rabinowitz, Periodic solutions for some forced singular Hamiltonian systems, from: "Analysis, et cetera", Academic Press, Boston (1990) 521-544 MR1039360

[28] J Roitberg, The Lusternik-Schnirelmann category of certain infinite CW-complexes, Topology 39 (2000) 95-101 MR1710994

[29] H Scheerer, D Stanley, D Tanré, Fibrewise construction applied to LusternikSchnirelmann category, Israel J. Math. 131 (2002) 333-359 MR1942316

[30] H Scheerer, M Stelzer, Fibrewise infinite symmetric products and $M$-category, Bull. Korean Math. Soc. 36 (1999) 671-682 MR1736612

[31] P A Schweitzer, Secondary cohomology operations induced by the diagonal mapping, Topology 3 (1965) 337-355 MR0182969

[32] S Shelah, Infinite abelian groups, Whitehead problem and some constructions, Israel J. Math. 18 (1974) 243-256 MR0357114 
[33] D Stanley, On the Lusternik-Schnirelmann category of maps, Canad. J. Math. 54 (2002) 608-633 MR1900766

[34] J Strom, Decomposition of the diagonal map, Topology 42 (2003) 349-364 MR1941439

[35] R M Switzer, Algebraic topology - homotopy and homology, Classics in Mathematics, Springer, Berlin (2002) MR1886843

[36] G W Whitehead, Elements of homotopy theory, Graduate Texts in Mathematics 61, Springer, New York (1978) MR516508

Department of Mathematics, Northwestern University

Evanston, IL 60208-2730, USA

Department of Mathematics, Dartmouth College

Hanover, NH 03755-3551, USA

Department of Mathematics, Western Michigan University

Kalamazoo, MI 49008, USA

rnendorf@math.northwestern.edu, nicholas.scoville@dartmouth.edu, jeff.strom@wmich.edu

Received: 5 January 2006 